\title{
Nachhaltigkeitsorientierte Weiterentwicklung von Industrie- und Gewerbeparks
}

\author{
Thomas Sterr
}

Online publiziert: 07. November 2012

(C) Springer-Verlag Berlin Heidelberg 2012

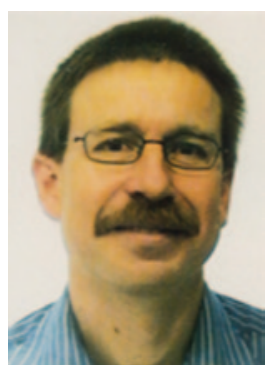

Thomas Sterr

Als 1989 im Industriegebiet der dänischen Mittelstadt Kalundborg von einer Projektgruppe von Gymnasiasten die Existenz einer sogenannten ,industrieller Symbiose“ entdeckt wurde ${ }^{1}$ und in den USA mit einem Artikel von Frosch/Gallopoulos der erste wissenschaftliche Artikel zur Idee ökoindustrieller Systeme erschien ${ }^{2}$, schlug de facto fast gleichzeitig an zwei Stellen der Welt die Stunde null für eine völlig neue, systemische Betrachtung industrieller Gebiete und deren material- und energiewirtschaftlicher Vernetzungspotenziale. Mit der Vorstellung der „Industriellen Symbiose von Kalundborg“ auf der vielbeachteten „Konferenz der Vereinten Nationen für Umwelt und Entwicklung“ (UNCED) im Juni 1992 in Rio de Janeiro erlangte die Idee quasi über Nacht weltweite Bekanntheit und löste damit ein enormes wissenschaftliches Forschungsinteresse aus, das

\footnotetext{
${ }^{1}$ Schwarz $(1994,98)$ unter Bezugnahme auf einen dänischsprachigen Aufsatz von Kragh (1990).

${ }^{2}$ Frosch und Gallopoulos (1989).
}

T. Sterr $(\square)$

IUWA-Institut für Umweltwirtschaftsanalysen Heidelberg e. V., Tiergartenstraße 17, 69121 Heidelberg, Deutschland

E-Mail: sterr@iuwa.de nicht nur die Erfolgsprinzipien des Phänomens Kalundborg systematisch zu identifizieren suchte, sondern gleichzeitig auch den weltweiten „Sternenhimmel“ an Industriegebieten mit Hilfe der neuen Brille abzusuchen begann. Der Gedanke, neue Industriegebiete künftig bereits als in ihrer Planungsphase sog. „Eco-industrial parks“ EIP zu designen oder bestehende zu „Zero Emissions Parks“ weiterzuentwickeln, verbreitete sich rasch und mit großer Euphorie, die sich alsbald in entsprechenden Projektvorhaben niederschlug ${ }^{3}$.

Heute, zwanzig Jahre nach Rio lässt sich nüchtern feststellen, dass die Umsetzung ökosystemarer Designs von „greenfield developments“ trotz des „erweiterten Bewusstseinshorizonts" und der hieraus abgeleiteten systematisierbaren Weiterentwicklungsmöglichkeiten deutlich hinter den anfänglichen Erwartungen zurückblieb, dass jedoch fast überall, wo firmenübergreifende Kommunikation angeregt werden konnte, auch neue Synergiepotenziale entdeckt und vielfach auch ausgeschöpft werden konnten. Dass die Erfolgsgeschichte von Kalundborg bis zum heutigen Tage anhält, ist eine Tatsache, dass sie aufgrund der Einzigartigkeit ihrer Entwicklungsbedingungen jedoch kaum direkt nachgeahmt werden kann, darf heute ebenfalls als gesicherte Erkenntnis gelten Entsprechende Dokumentationen und Anregungen siehe bspw. Holländer et al. (2005), Lowe (2001), Salonen (2010) oder Sterr (2003). Das heißt nicht, dass es in Deutschland heute nicht ähnlich intensive Vernetzungen formal selbständiger Firmen gäbe wie im Falle von Kalundborg, aber die „Story“ dahinter ist eine grundsätzlich andere. So wie bspw. der Produktionsstandort der BASF in Ludwigshafen durch seine ,integrierte Verbundproduktion" gegenüber einem theoretischen Referenzszenario, das der durchschnittlichen Verteilung von Chemiestandor-

\footnotetext{
${ }^{3}$ Siehe v. a. die zahlreichen EIP-Projekte in den USA der 90er Jahre.
} 
ten in Deutschland entspricht, ca. 500 Mio. $€ / \mathrm{Jahr}$ spart $^{4}$, profitieren auch mehrere inzwischen atomisierte Chemieunternehmen von der Ver- und Entsorgungsvernetzung ihrer ehemaligen Werksteile. Ein Beispiel hierfür bietet der Produktionsstandort des ehemaligen Chemieriesen Höchst bei Frankfurt, an dem heute in 90 selbständigen Unternehmen produziert wird ${ }^{5}$, während der ehemalige Geschäftsbereich Ver- und Entsorgung der Höchst Werke heute als selbstständiger Standortbetreiber auftritt. Die heutige Infraserv $\mathrm{GmbH} \&$ Co. Höchst KG ist in ihrem umfassenden technischen und organisatorischen Dienstleistungsangebot inzwischen so erfolgreich, dass sie nicht nur die Nachfolgeunternehmen der ehemaligen Höchst $A G$ bedient, sondern auch weit darüber hinaus technisch und beratend tätig ist (Infraserv 2012). Ganz ähnlich kann die Rolle der Firma Currenta GmbH \& Co. OHG beschrieben werden, die als Joint Venture der Bayer AG und der Lanxess AG in Deutschland drei Chemiestandorte betreibt ${ }^{6}$. Überspitzt formuliert könnte man demnach die These aufstellen, dass die bedeutendsten öko-industriellen Symbiosen in Deutschland nicht etwa evolutorisch auf Basis einer systematischen Ausnutzung von Synergiepotenzialen zwischen verschiedenen räumlich benachbarten Unternehmen aufgebaut wurden, sondern als Folge der Zerstückelung ehemaliger Chemieriesenentstanden.

Wenn wir heute in Länder wie China schauen, dann stellen wir fest, dass das firmenverbindende Leistungsspektrum industrieparkbezogener Ver- und Entsorgungsdienstleister auch dort mit großer Bewunderung verfolgt wird und deutsche Chemieparkbetreiber (wie die beiden oben genannten) auch gerade auch für die Weiterentwicklung dortiger Chemieparks als vorbildlich gelten. Tatsächlich lehrt gerade die Erfolgsgeschichte lehrt moderner Industriestandortbetreiber, dass das systemische Verständnis von Industrie- und Gewerbeparks enorme Effizienzsteigerungspotenziale bietet, dass diese aber nicht nur im Bereich stoff- und energiewirtschaftlicher Output-Input-Kombinationen sowie gemeinsamer Ver- und Entsorgungsinfrastruktur zu suchen sind, sondern mit systemadäquaten Managementansätzen verbunden werden können ${ }^{7}$, die sehr viel breiter angelegt sind. Ein solches über die Stoff- und Energiewirtschaft hinausgehendes Industriestandortmanagement vermag nicht nur die buchhalterisch sichtbaren Kosten zu drücken, sondern auch schwieriger quantifizierbare soziale Funktionen mit zu erfüllen und den Industriestandort als menschlichen

\footnotetext{
${ }^{4}$ BASF Vortrag (PPT) zur Messung von Verbundeffekten von Wolpert, April 2009 (BASF/Wolpert 2009).

${ }^{5} \mathrm{http}: / /$ www.industriepark-hoechst.com/.

${ }^{6}$ Leverkusen, Dormagen, Krefeld-Uerdingen; siehe http://www.bayer. de/de/currenta.aspx (Currenta 2012).

${ }^{7}$ Siehe hierzu insbesondere der Aufsatz von Reinfeldt in dieser Ausgabe.
}

Lebens- und Schaffensraum insgesamt zu befördern und nach außen hin sichtbar werden zu lassen. Auch das brandaktuelle Thema „Ressourceneffizienz“ erhält hierdurch einen wesentlich breiteren Anspruch.

Aber werfen wir nun einfach einmal einen etwas genaueren Blick auf die Erkenntnisse und Möglichkeitsräume von heute und lassen uns anhand verschiedener Expertenmeinungen zu neuen Ideen, Konzepten und Strategien im Sinne einer nachhaltigkeitsorientierten Weiterentwicklung von Industrie- und Gewerbeparks in verschiedenen Teilen der Welt anregen.

\section{Literatur}

BASF/Wolpert (2009) Ökoeffizienz-Analyse und Vorteile des Verbundes (am Beispiel der BASF); PPT zu einem Fachvortrag im Rahmen eines Delegationsbesuches bei der BASF in Ludwigshafen (Dokument vom April 2009)

Currenta (2012). http://www.bayer.de/de/currenta.aspx. Zugegriffern: 14. Okt. 2012

Frosch RA, Gallopoulos NE (1989) Strategien für die Industrieproduktion. Spekt Wissensc 11:126-144

Holländer F, Salonen T, Wu Ch, Geng Y (2005). Sustainable management of industrial parks - Proceedings of the German-Chinese Workshop, October 12-16, 2004 in Leipzig, Germany. Logos publishers, Berlin

Infraserv (2012). http://www.industriepark-hoechst.com/. Zugegriffen: 14. Okt. 2012

Lowe E (2001) Eco-industrial handbook for Asian developing countries. Downloadmöglichkeit über http://indigodev.com/ ADBHBdownloads.html

Salonen T (2010) Strategies, structures, and processes for network and resources management in industrial parks: the cases of Germany and China: Diss. Leipzig University, Lohmar publishers, Leipzig

Schwarz E (1994) Unternehmensnetzwerke im Recyclingbereich (Diss). Gabler, Wiesbaden

Sterr Th (2003) Industrielle Stoffkreislaufwirtschaft im regionalen Kontext: Betriebswirtschaftlich-ökologische und geographische Betrachtungen in Theorie und Praxis. Springer, Berlin

Von Hauff M, Isenmann R, Müller-Christ G (2012) Industrial ecology management. Nachhaltige Entwicklung durch Unternehmensverbünde 